\title{
News and Expectations in Financial Markets: An Experimental Study
}

\author{
Gordon Douglas Menzies ${ }^{1}$ \\ School of Finance and Economics, University of Technology, Sydney \\ CAMA, Australian National University \\ Daniel John Zizzo ${ }^{2}$ \\ School of Economics and CBESS, University of East Anglia \\ CAMA, Australian National University
}

\begin{abstract}
We consider an experimental setting where agents receive one stylized piece of information at a time about the value of a financial asset. We find that Knightian uncertainty about the prior distribution of true financial asset values does not hamper decision making by agents and markets. On a mean squared error criterion, Bayesian updating is closer than simple averaging in predicting market prices and individual bids and offers, even in treatments with uncertainty where Bayesian updating should not be feasible given the limited information set. Bayesian updating also outperforms adaptive expectations in relation to market prices.

Keywords: uncertainty, expectations, information, framing. JEL Classification Codes: C91, D83, D84, F31, G12.
\end{abstract}

\footnotetext{
${ }^{1}$ Tel: +61-2-95147728; fax +61-2-95147711. Email address: gordon.menzies@uts.edu.au

2 Tel: +44-1603-593668; fax: +44-1603-456259. Email address: d.zizzo@uea.ac.uk

We are grateful to the British Academy for financial support, to participants to presentations in Lyon, Norwich and Sydney for helpful feedback, and to James Watson, Nicole Huang, Kei Tsutsui and Maya Elliott for research assistance. The usual disclaimer applies. The experimental instructions can be found in an online appendix.
} 


\section{Introduction}

This paper considers a simple experimental setting where agents receive one stylized piece of information at a time about the value of a financial asset. We consider whether, in a market setting, assets will be priced differently if there is only limited information about the nature of the financial asset. Recent events bear out the centrality of shares and foreign exchange for economic performance and for the transmission of global shocks. We therefore consider the pricing of shares and foreign exchange, after each piece of information is received. Our specific question is: does Bayesian updating, which requires full information about the financial asset, perform worse than employing the maximum likelihood estimator, which does not?

To expand, the key question we try to answer is whether the essential uncertainty (as opposed to risk, following Knight's, 1921, distinction) intrinsic in the information set available to traders makes them update their beliefs differently, resulting in a different trajectory of market prices. We isolate the effect of uncertainty by having a treatment where the distribution of true financial asset value is very limited, and one in which it is fully known.

This is of interest because both individual choice and market experiments, (as in, Ellsberg, 1961, and Sarin and Weber, 1993) show that subjects dislike ambiguity, i.e. Knightian uncertainty. While our paper is not about ambiguity aversion per se, this literature shows intrinsic ambiguity might matter when agents process information and trade as a result.

Identifying whether uncertainty matters is important for another, and more general, reason: if agents do not have sufficient information about the prior distribution of the value of the financial asset (including the standard deviation), Bayesian signal extraction is not feasible in principle on the part of rational expectations agents, unless they append 'educated guesses' 
about the prior distribution to their inference procedure. If they refrain from these auxiliary assumptions, the rational procedure is maximum likelihood estimation (MLE) based on an average of the signals received. Therefore, how we model expectation formation is potentially a function of how agents process the intrinsic ambiguity in the market environment.

Our key and surprising finding is that Bayesian models of expectation formation tend to outperform the corresponding MLE models, even in the no prior distribution knowledge treatments. We perform our experiment in two different frames for the market (an exchange rate market frame vs. stock market frame), as we are conscious that subjects can sometimes more or less close to rational choices depending on the frame used (e.g., Cosmides and Tooby, 1992), and we find that the key finding of outperformance by Bayesian expectations is robust between frames. ${ }^{3}$

Section 2 presents the theoretical setup and solutions under different expectations regimes. Sections 3 and 4 describe the experimental design and results, while section 5 concludes.

\section{Theoretical Setup and Solutions for Different Expectation Assumptions}

\subsection{Setup}

The theoretical setup is one in which agents must infer the value of a single parameter drawn from a distribution:

$$
\lambda \sim\left(\Lambda, \sigma^{2}\right)
$$

In period zero, agents trade without any sample information. Thereafter, they trade on the basis of a sequence of noisy signals $\lambda+e_{t} e_{t} \sim\left(0, \sigma_{e}^{2}\right)$ which contain information about this unknown parameter:

\footnotetext{
${ }^{3}$ Our analysis also includes a standard adaptive expectations (ADE) specification, with a weight $\beta$ assigned to the previous period's observation in forming the current expectation. ADE are included as they are often purported to be good data descriptors despite a lack of theoretical microfoundation (e.g., Mankiw, 2001) or even any model consistency requirement.
} 


$$
\begin{aligned}
& x_{1}=\lambda+e_{1} \\
& x_{2}=\lambda+e_{2} \\
& \cdots \cdots \cdots \cdots . . . \\
& x_{t}=\lambda+e_{t}
\end{aligned}
$$

and they must guess $\lambda$ after each $\mathrm{x}_{\mathrm{i}}$ is revealed. We now outline two solutions.

\subsection{The Maximum Likelihood Solution (MLE Expectations)}

From period one onwards, the simplest solution is simply to average the signals:

$$
\hat{\lambda}_{t}^{M}=\frac{\sum_{i=1}^{t} x_{i}}{t}=\lambda+\frac{\sum_{i=1}^{t} e_{i}}{t}
$$

Conditional on $\lambda$, this has all the desirable least-squares and maximum likelihood properties

$$
\begin{aligned}
& E\left(\left.\hat{\lambda}_{t}^{M}\right|_{\lambda}\right)=\lambda \\
& V\left(\left.\hat{\lambda}_{t}^{M}\right|_{\lambda}\right)=\frac{\sigma_{e}^{2}}{t}
\end{aligned}
$$

and the Gauss-Markov theorem holds. ${ }^{4}$

Nevertheless, the estimator does not use all information. There is prior information on the distribution of $\lambda$, namely $\Lambda$ and $\sigma^{2}$.

\subsection{The Bayesian Signal Extraction Solution (Bayesian expectations)}

Consider a class of solutions of the form:

$$
\begin{aligned}
& \hat{\lambda}_{1}^{B}=\theta \Lambda+(1-\theta) x_{1} \\
& \hat{\lambda}_{t}^{B}=\theta \hat{\lambda}_{t-1}^{B}+(1-\theta) x_{t}
\end{aligned}
$$

\footnotetext{
${ }^{4}$ That is, conditional on $\lambda$ it is the minimum variance estimator among the class of all linear unbiased estimators.
} 
In other words, the first guess is based on a weighted average of the known mean of $\lambda$, namely $\Lambda$, and the signal, with the optimal weights to be determined presently. Thereafter, agents use a recursion with the same weights.

In general, optimal weights for (2) depend on the time horizon for which signals are available. But the solution requires numerical optimization, so we derive the optimal weight $\theta$ for the first period, and assume it prevails in the solution. ${ }^{5}$ Consider the first period:

$$
\begin{aligned}
\begin{aligned}
& \hat{\lambda}_{1}^{B} \quad \theta \Lambda+(1-\theta) x_{1} \\
&=\theta \Lambda+(1-\theta)\left(\lambda+e_{1}\right) \\
&=(1-\theta) e_{1}+\lambda+\theta(\Lambda-\lambda) \\
& \Rightarrow \hat{\lambda}_{1}^{B}-\lambda \quad=\quad(1-\theta) e_{1}+\theta(\Lambda-\lambda)
\end{aligned}
\end{aligned}
$$

We now seek $\theta$ that will minimize the expected value of the mean squared error (MSE). When taking the expectation, we do not condition on $\lambda$, because we are seeking an optimal weight that takes account of its distribution. We first derive the unconditional mean and variance (V[.]):

$$
\begin{aligned}
E\left(\hat{\lambda}_{1}^{B}-\lambda\right) & =E(1-\theta) e_{1}+\theta E(\Lambda-\lambda)=0 \\
V\left(\hat{\lambda}_{1}^{B}-\lambda\right) & =V\left((1-\theta) e_{1}\right)+V(\theta(\Lambda-\lambda)) \\
& =\left((1-\theta)^{2} \sigma_{e}^{2}+\theta^{2} \sigma^{2}\right)
\end{aligned}
$$

We now minimize the MSE:

\footnotetext{
${ }^{5}$ Equation (2) can be written as $(1-\theta)\left[e_{t}+\theta e_{t-1}+\ldots+\theta^{t-1} e_{1}\right]+\lambda+\theta^{t}(\Lambda-\lambda)$. An Optimal $\theta$ which minimize $\sum_{i=1}^{t} \mathrm{E}\left(\hat{\lambda}_{t}^{B}-\lambda\right)^{2} / \mathrm{t}$, as in the text, requires a numerical solution, as it is a function of $\mathrm{t}$. When this expression is optimized for $\mathrm{t}=8$, as in our experiment, the optimal $\theta$ is 0.71 .
} 


$$
\begin{aligned}
E\left[\left(\hat{\lambda}_{1}^{B}-\lambda\right)^{2}\right] & =V\left(\hat{\lambda}_{1}^{B}-\lambda\right)+\left\{E\left(\hat{\lambda}_{1}^{B}-\lambda\right)\right\}^{2} \\
& =\left((1-\theta)^{2} \sigma_{e}^{2}+\theta^{2} \sigma^{2}\right)+0 \\
\frac{d E\left[\left(\hat{\lambda}_{1}^{B}-\lambda\right)^{2}\right]}{d \theta} & =-2(1-\theta) \sigma_{e}^{2}+2 \theta \sigma^{2}=0 \\
\Rightarrow \quad \theta \quad & =\frac{\sigma_{e}^{2}}{\sigma_{e}^{2}+\sigma^{2}}
\end{aligned}
$$

Our solution is called $\hat{\lambda}_{1}^{B}$ because this is a classic Bayesian signal extraction solution. ${ }^{6}$

\subsection{Adaptive Expectations (ADE)}

Under adaptive expectations, agents are conservative in the sense that they anchor their estimate of $\lambda$ on the last period's price of the financial asset, called $\mathrm{p}_{\mathrm{t}-1}$. Thus, $\hat{\lambda}_{t}^{A}=\beta p_{t-1}+$ error. There is no theoretical value for $\beta$, so it must be estimated. It is obtained by Least-Squares where we proxy $\hat{\lambda}_{t}^{A}$ with the current price $\mathrm{p}_{\mathrm{t}}$. That is, we run the regression $p_{t}=\beta p_{t-1}+$ error

\section{Experimental Design}

The experiment was conducted between January and June 2008 at the university of the second author. ${ }^{7}$ Apart from the experimental instructions and a control questionnaire, the experiment was fully computerized. A total of 240 subjects participated in the 48 sessions: five subjects participated to each session, and they participated to one of four treatments (discussed below), giving a total of 12 independent observations per treatment. Subjects were randomly seated in the laboratory. Computer terminals were partitioned to avoid communication by visual or verbal means. Subjects read the experimental instructions and answered a control questionnaire before being allowed to proceed with the tasks.

\footnotetext{
${ }^{6}$ One subtle difference is that we do not require normal distributions, even though, in fact, we do use normal distributions for our experiment.

${ }^{7}$ The experimental instructions are provided in electronic appendix A.
} 
Experimental supervisors individually advised subjects with incorrect answers in the questionnaires.

The experiment lasted up to 2 hours and was divided into four stages, each divided in turn in 9 trading periods. The periods were divided into period 0 , when agents didn't receive a signal, and periods 1 to 8 , where they did, as described below. The assets traded were either foreign currency (in an exchange rate frame) or shares (in a financial frame). Subjects did not know exactly what the intrinsic value $\lambda$ of the financial asset was, though they knew that assets retained the same value $\lambda$ throughout each stage though not across stages.

In the 'limited prior knowledge' treatments, subjects only knew that the financial asset value was drawn from a prior symmetric distribution with mean 1 (i.e., that on average a unit of the financial asset could be transferred one-for-one into pounds at the end of the stage), but they did not know the exact shape or, crucially, standard deviation of the prior distribution. As a result, Bayesian updating was not feasible unless we assume that subject behaved as if they made auxiliary assumptions. Technically, averaging is the only feasible solution.

In the 'prior knowledge' distribution, subjects were provided information about the prior distribution in the form of a 'table of frequencies' (effectively, a histogram); information was provided as a table of frequencies rather than of probabilities since it is known that subjects process information more efficiently in terms of the former than in term of the latter (e.g. Hertwig and Gigerenzer, 1999). As a result, subjects could infer the standard deviation of the prior distribution, and Bayesian updating become feasible. The distribution actually used was the same in both the 'limited prior knowledge' and the 'prior knowledge' treatments. It was normal with mean 1 and standard deviation 0.25: only the extent of knowledge about the distribution differed across treatments.

Overall, the experiment had a $2 \times 2$ factorial design crossing the frame employed (stock market vs. exchange rate market) and the knowledge about the prior distribution. We had four 
treatments overall, $\mathrm{AE}$ (knowledge, exchange rate frame), BE (limited knowledge, exchange rate frame), AS (knowledge, stock market frame) and BS (limited knowledge, stock market frame).

\section{(Insert Table 1 about here.)}

At the beginning of each period subjects received 10 units of each of two assets (foreign and home currency in one frame, cash and shares in the other frame), with only imperfect information about their intrinsic value $\lambda$ at which the financial assets would ultimately be converted to pounds. The information that subjects had differed depending on the treatment, and is described below, but was common across subjects of any given session. Trade occurred according to a Walrasian clearinghouse auction mechanism, with each subject being asked to provide a buying price and a selling price. The excellent efficiency properties of Walrasian auctions are well-known, and close to that of double auctions (e.g., Smith et al., 1982). ${ }^{8}$ Trade could only take place using the endowments received at the beginning of the relevant trading period. A generic experimental session is represented in the diagram below.

\section{(Insert Figure 1 about here.)}

At the beginning of each stage a new value for the true value $\lambda$ of the financial asset was drawn applicable to the stage, and subjects knew that. From the start of period 1 onwards, subjects received an independent noisy signal about the value of the financial asset of the kind discussed in section 2, i.e. of the form $\lambda+e_{t} e_{t} \sim\left(0, \sigma_{e}^{2}\right), \mathrm{t}=1$ to 8 . Subjects received a table of frequencies illustrating the (normal) distribution of the signal around its true value (see the appendix). In AE and AS, they were also shown a table of frequencies of $\lambda$.

\footnotetext{
${ }^{8}$ A Walrasian auction has the additional advantages of making certain that an equilibrium market price (exchange rate) would be formed (as bids and offers were required on the part of each trader), that there was a single value of the exchange rate per stage and that all trade was completed, with all bids and offers being elicited, within a shorter amount of time than a double auction (in an experiment that could already last over two hours, time was very much at a premium).
} 
In all treatments, to help with understanding, subjects also received an example sheet showing examples of how the economy may work in each stage. At the end of each stage, the units of the financial asset obtained were converted into home currency (exchange rate frame) or cash (stock market frame) using the true value $\lambda$. At the end of the experiment, one period was chosen at random by the computer and each unit of home currency or cash earned in that period (whether directly or after the conversion using $\lambda$ ) was translated into U.K. pounds at the rate of 1 pound per unit. Mean experimental payment were 22-23 UK pounds (roughly 40 US dollars) per subject for between $1 \frac{1 / 2}{2}$ and 2 hours of work.

\section{Experimental Results ${ }^{9}$}

\subsection{General Results}

Figure 2 shows examples of financial asset price dynamics in one session each from each treatment. ${ }^{10}$ Our focus below is on periods 1 through 8 , since predictions of Bayesian and MLE expectations can only diverge after agents receive signals, and ADE predictions can only be identified when there is a lag. ${ }^{11}$ For ADE, we estimate the lagged dependent variable coefficient using least-squares on market prices, individual buying prices, and individual selling prices). The coefficients for the different treatments, denoted $\beta$, are given in Table 2 .

(Insert Figure 2 and Table 2 about here.)

Subjects generally appeared to have a good understanding of the experiment, and financial asset prices were almost always in the 0.5 to 1.5 range with a mode around 1 , which is what we would expect with our $N \sim(1,0.25)$ distribution of true financial asset values (see Figure 3).

(Insert Figure 3 about here.)

\footnotetext{
${ }^{9}$ All $\mathrm{P}$ values reported in this paper are two tailed. All tests are at the session level to guarantee the non independence of observations.

${ }^{10}$ Electronic appendix B contains similar graphs for all sessions.

${ }^{11}$ That being said, no result would change if we were to include period 0 .
} 
Subjects traded financial asset units $62 \%$ of the times (between $59 \%$ and $64 \%$ depending on the treatment). Table 3 includes average, variance, skewness and kurtosis of financial asset prices, individual buying prices, individual selling prices and quantities traded in all four treatments. We provide both mean and median information, since, particularly in relation to selling prices and buying prices, median information helps filter out noisy outliers. Not surprisingly, market prices tend to be more stable than either selling prices or buying prices. That being said, a comparison of AE and AS vs. BE and BS shows that the introduction of uncertainty had no statistically significant effect not only on market prices, but also on selling prices, buying prices and quantity traded in Mann Whitney tests; the closest we get to significance is in market prices being marginally higher under uncertainty (1.001) than under full information (0.967), which yields $\mathrm{P}=0.091$, but Table 3 shows that this small effect appears frame dependent. The frame manipulation appears to matter more, with the mean market price $(\mathrm{P}=0.025)$, quantity bought $(\mathrm{P}=0.041)$, skewness and kurtosis of quantity bought $(\mathrm{P}<0.001$ in both cases) all resulting in statistically significant tests. Market prices and quantities traded are slightly lower under a stock market frame than under an exchange rate frame, with the distribution of quantities bought being however a little more skewed towards higher values and kurtosis being marginally higher.

RESULT 1. Uncertainty generally does not affect the average, variance, skewness and kurtosis of the distributions of market prices, buying prices, selling prices and quantities traded. Framing matters more than uncertainty does.

\subsection{Comparing the goodness of fit of different classes of models}

We now use the sum of squared errors (SSE) between predicted and observed exchange rates to compare the goodness of fit of the different classes of models: Bayesian, MLE and 
adaptive expectations. Figure 4 compares the goodness of fit of different expectation models in predicting financial asset values.

(Insert Figure 4 about here.)

Bayesian expectations consistently have better predictive power than the simple averaging implied by MLE expectations, as reflected in a lower sum of squared errors in all treatments, including the $\mathrm{BE}$ and $\mathrm{BS}$ treatments with limited knowledge about the prior distribution. This superiority is unequivocally confirmed in Wilcoxon tests $(\mathrm{P} \leq 0.005)$, and is also confirmed if we look at SSE in relation to individual buying or selling prices. Across treatment differences in SSE are never statistically significant in Kruskal Wallis or Mann Whitney tests.

RESULT 2. Bayesian expectation models have better predictive power than MLE expectation models in all treatments, including those with limited knowledge about the prior distribution.

The SSE in relation to adaptive expectations is lower than that in relation to Bayesian updating in treatment $\mathrm{AE}$, but not statistically significantly so. It is higher in the other treatments. Overall, at the market level there is moderately strong evidence that the Bayesian models outperform adaptive expectations, $(\mathrm{P}=0.055$ in a Wilcoxon test $)$. These results contrast with the superiority of adaptive expectations over Bayesian updating at the level of individual buying and selling prices. There is a sense, therefore, that markets are "more rational' (in terms of Bayesian fit relative to adaptive expectations) than individuals.

RESUlt 3. At the individual level, adaptive expectations outperform Bayesian expectations. At the market level, Bayesian expectations globally outperform adaptive expectations. 


\section{Conclusion}

This paper began with a specific question: does Bayesian updating, which requires full information about the financial asset, perform worse than employing the maximum likelihood estimator, which does not? Our answer, which is a qualified 'no', has two sub-parts. The first is that explicit knowledge of the prior distribution of true financial asset values is not necessarily crucial for subjects to behave rationally. The second is that, in our setup, the Bayesian signal extraction framework describes behavior just as well without such knowledge as it did with it. Interestingly, allowing for estimating one degree of freedom in an adaptive expectation model did not enable adaptive expectations to outperform Bayesian signal extraction models at the level of market prices.

The qualification is that our subjects do behave a little differently when trading foreign exchange and shares. In particular, they trade higher quantities of the former. While the main results are robust across the two frames, it might be interesting, and prudent, to further explore the differences in belief formation in these two markets before declaring generality.

Our results might seem to be out of step with the groundswell against rational expectations as a modelling tool, particularly since the Global Financial Crisis (Akerlof and Shiller 2009). In fact, we would not wish to deny that uncertainty does matter in a number of contexts. However, if our results stand the scrutiny of further testing they suggest that there might be at least some market setups where economists may be able to model behavior as if agents know the prior distribution when they trade shares or foreign exchange.

\section{References}

Akerlof, G. A. \& Shiller, R. J. (2009) Animal Spirits: How Human Psychology Drives the Economy, and Why It Matters for Global Capitalism, Princeton: Princeton University Press.

Cosmides, L., Tooby, J. (1992). Cognitive adaptations for social exchange. In J.H. Barkow, L. Cosmides \& J. Tooby (Eds.), The adapted mind: Evolutionary psychology and the 
generation of culture (pp. 163-229). New York and Oxford: Oxford University Press.

Ellsberg, D. (1961). Risk, ambiguity, and the Savage axioms. Quarterly Journal of Economics, 75, 643-669.

Hertwig, R., \& Gigerenzer, G. (1999). The 'conjunction fallacy' revisited: How intelligent inferences look like reasoning errors. Journal of Behavioral Decision Making, 12, 275305.

Knight, F. H. (1921). Risk, Uncertainty, and Profit. Boston, MA: Hart, Schaffner \& Marx. http://www.econlib.org/LIBRARY/Knight/knRUP1.html

Mankiw, N. G. (2001). The inexorable and mysterious tradeoff between inflation and unemployment. Economic Journal, 111, C45-61.

Sarin, R. K., \& Weber, M. (1993). Effects of ambiguity in market experiments. Management Science, 39, 602-615.

Smith, V. L., Williams, A. W., Bratton, K. W., \& Vannoni, M. G. (1982). Competitive market institutions: Double auctions vs. sealed bid-offer auctions. American Economic Review, 72: $58-77$.

Figure 1. Timeline of Experimental Sessions

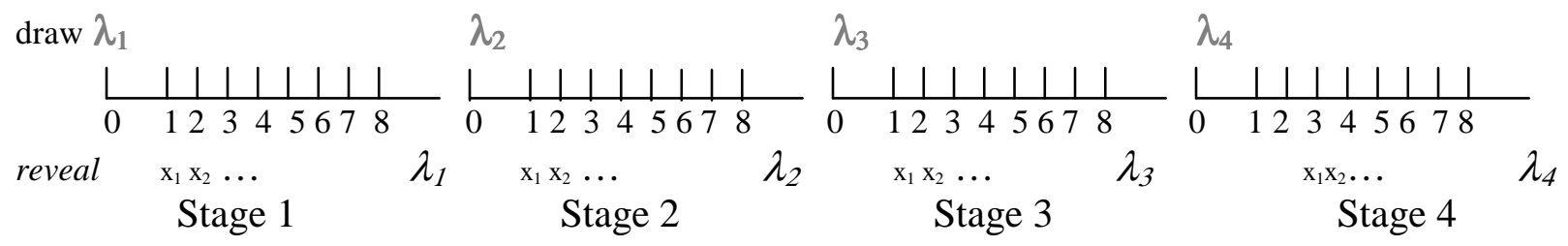




\section{Figure 2. Observed Prices and Predictions in Sample Sessions}
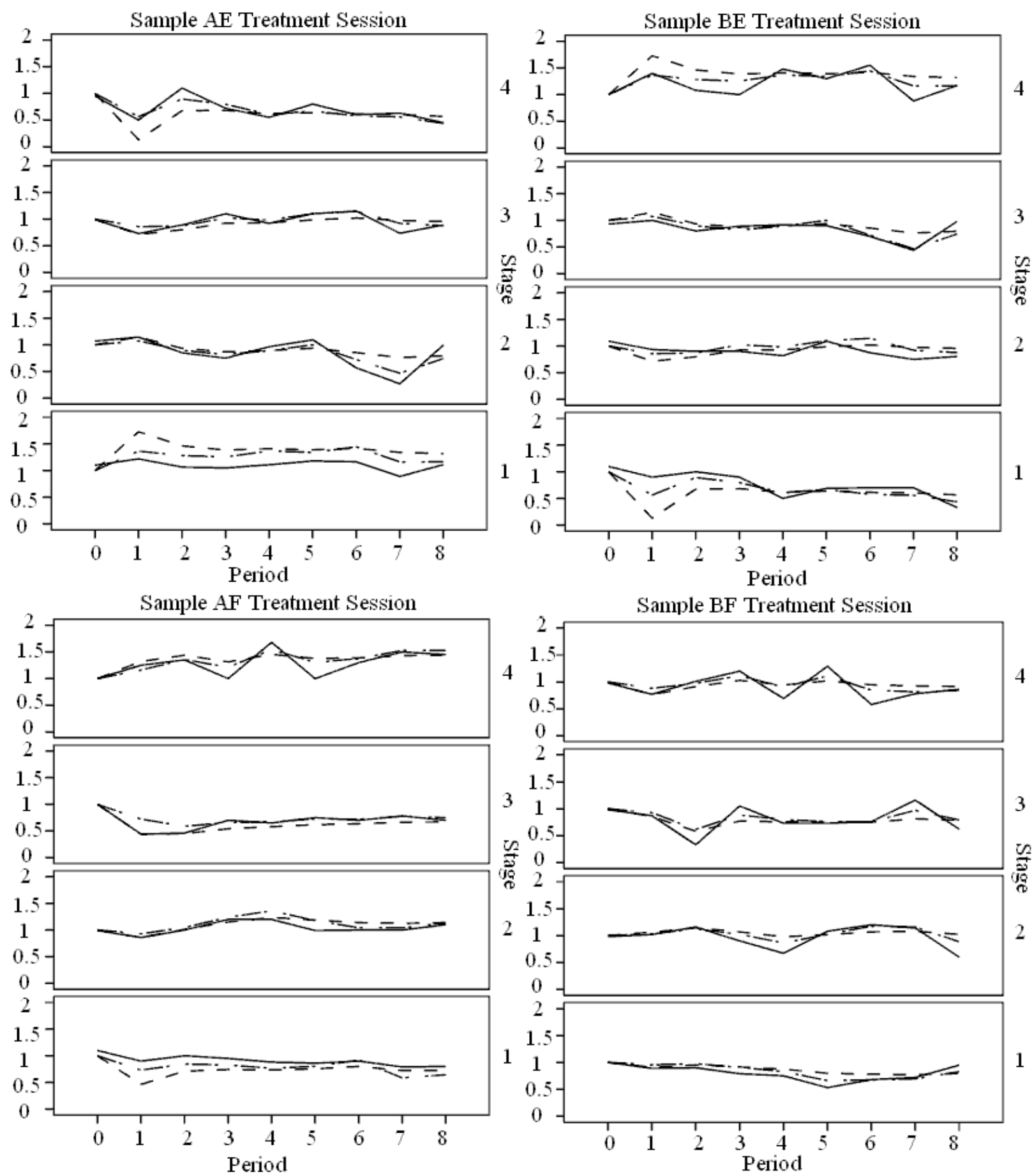

- Observed Price

- - Bayesian Expectation

- - MLE Expectation

Note: the adaptive expectations prediction is equal to the observed price in the previous period. 
Figure 3. Histograms of Financial Asset Values

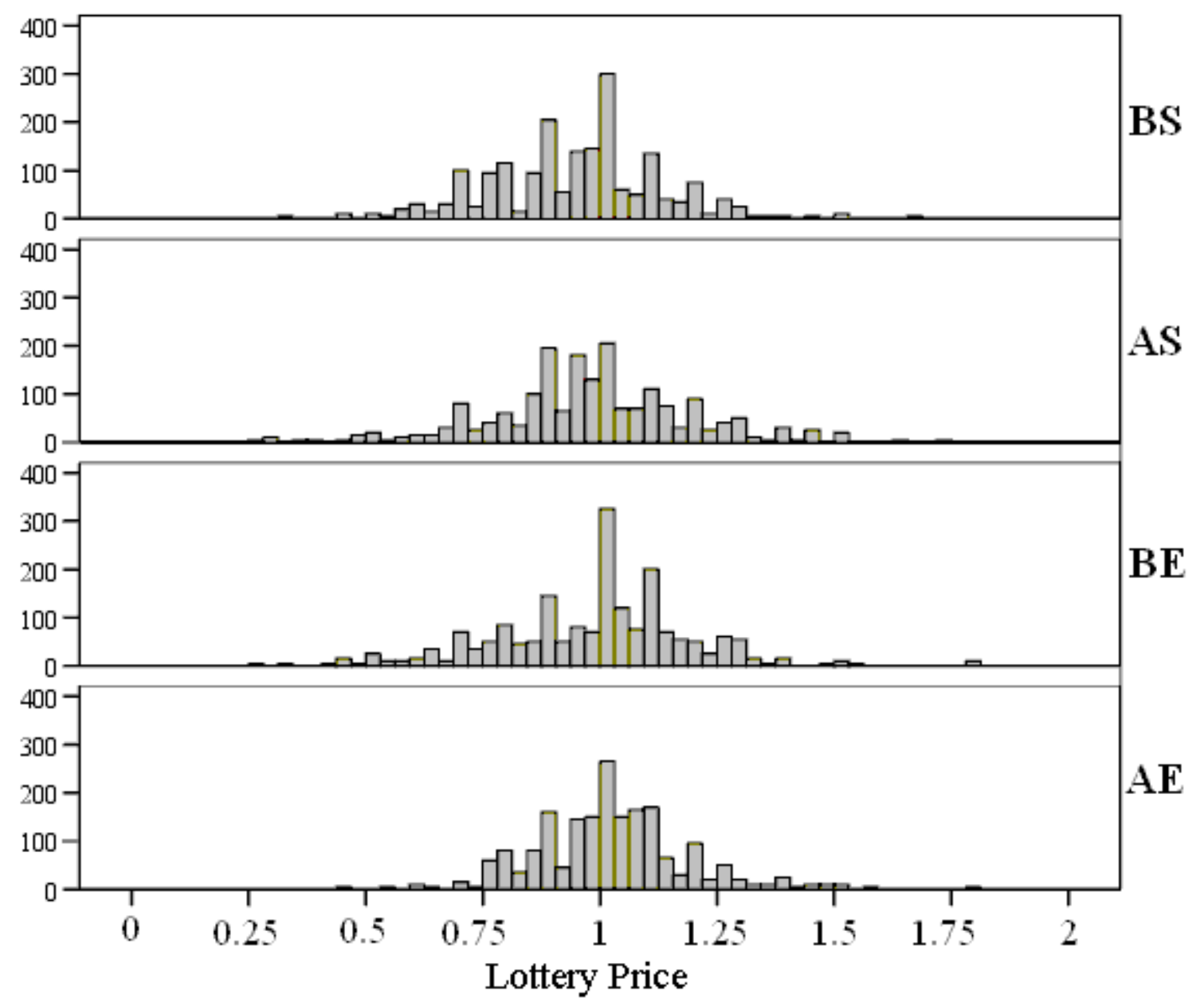

Note: Based on asset prices (i.e. price of foreign exchange or shares) in periods 1-8. 
Figure 4. Goodness of Fit of Different Expectation Models

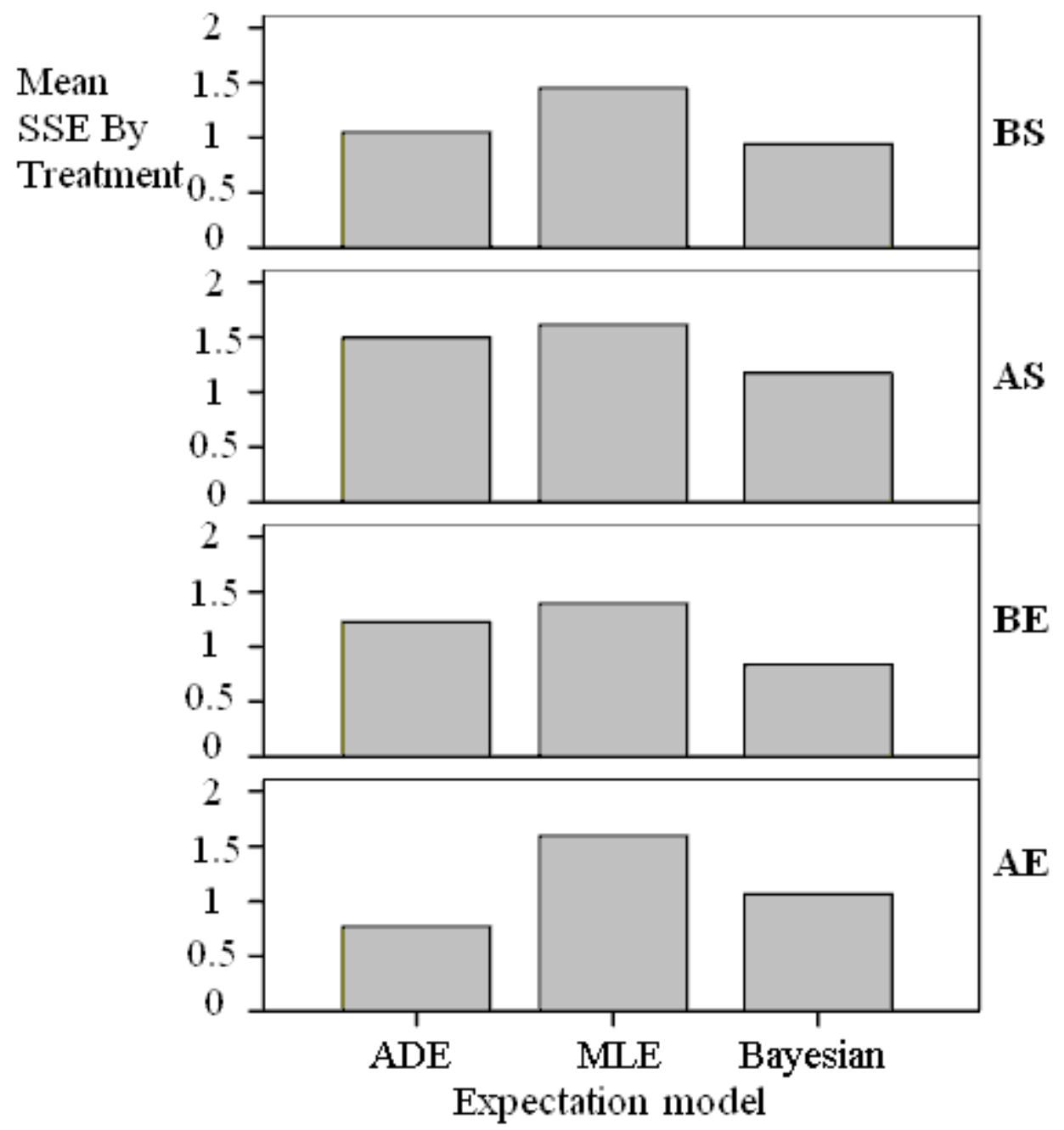

Notes: SSE: sum of squares error; AE: adaptive expectations; MLE: maximum likelihood expectations; Bayesian: Bayesian expectations. 
Table 1. Experimental Treatments

\begin{tabular}{ll}
\hline Treatment AE & Treatment BE \\
Knowledge of Prior Distribution, & No Knowledge of Prior Distribution \\
Exchange Rate Frame & Exchange Rate Frame \\
\hline Treatment AS & Treatment BS \\
Knowledge of Prior Distribution, & No Knowledge of Prior Distribution \\
Stock Market Frame & Stock Market Frame \\
\hline
\end{tabular}

Note: 12 sessions were run in each treatment.

Table 2. Estimated Mean $\beta$ Values by Type of Prices and Treatment

\begin{tabular}{ccccccc}
\hline \multicolumn{5}{c}{ Treatment } & & \\
& & AE & BE & AS & BS & Overall \\
\hline$\beta$ & Market prices & 0.987 & 0.971 & 0.965 & 0.974 & 0.974 \\
& Buying prices & 0.991 & 0.918 & 0.903 & 0.891 & 0.926 \\
& Selling prices & 0.932 & 0.899 & 0.884 & 0.904 & 0.905 \\
\hline
\end{tabular}

Notes: The adaptive expectations parameter $\beta$ was estimated, in each case, by minimizing the sum of squares error between actual and predicted values. 
Table 3. Distributions of Market Prices, Selling Prices, Buying Prices and Quantity Traded

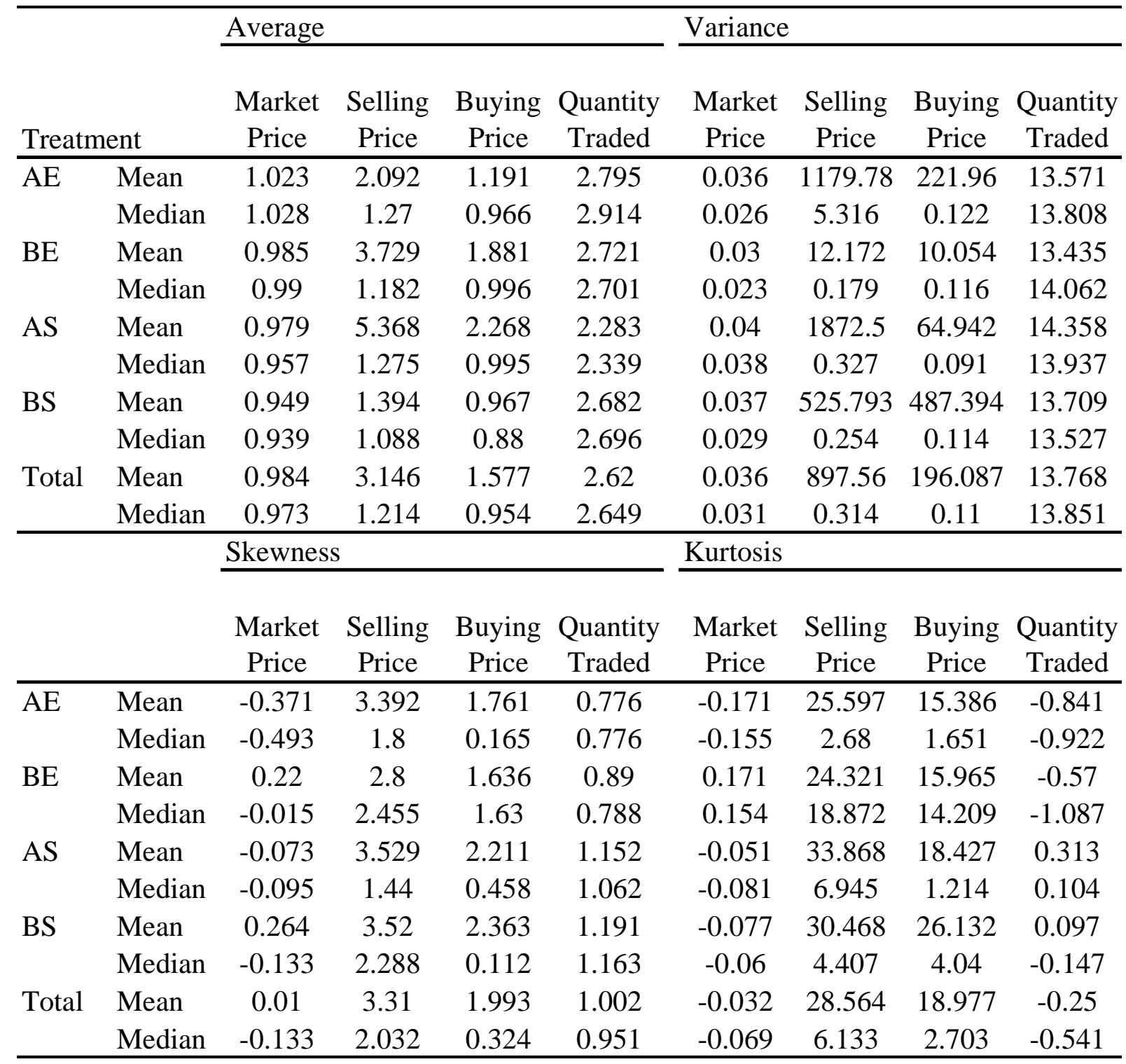

